\title{
INDEX TO VOLUME 74
}

\section{RESEARCH ANNOUNCEMENTS}

Akutowitz, E. J. On mean-periodicity, 698.

Allan, N. D. On the commensurability class of the Siegel modular group, 115.

Allen, H. P. and Ferrar, J. C. New simple Lie algebras of type $D_{4}, 478$.

Anshel, Michael and Clay, J. R. Planarity in algebraic systems, 746.

Arkowitz, Martin. A homological method for computing certain Whitehead products, 1079.

Arnold, Ludwig and Michalicek, Johannes. On the norm of stable measures, 364 .

Askey, Richard. Jacobi polynomial expansions with positive coefficients and imbeddings of projective spaces, 301.

Barr, Michael, A note on commutative algebra cohomology, 310.

Baumslag, Gilbert. More groups that are just about free, 752.

Beals, Richard. Global estimates for elliptic spectral functions and eigenvalues, 358.

, On eigenvalue distributions for elliptic operators without smooth coefficients. II, 1024.

Ben-Israel, A. and Charnes, A. On the intersections of cones and subspaces, 541.

Berens, H., Butzer, P. L. and Westphal, U. Representation of fractional powers of infinitesimal generators of semigroups, 191.

Berg, I. D. and Rubel, L. A. Densities on locally compact Abelian groups, 298.

Berger, C. A. and Coburn, L. A. C*algebras of translations and multipliers, 1008.

Berstein, Israel. Involutions with nonzero Arf invariant, 678.

Bhattacharya, R. N. Berry-Esseen bounds for the multi-dimensional central limit theorem, 285.

Binmore, K. G. and Katz, Melvin. A note on the strong law of large numbers, 941.

Blakely, G. R. Intrinsic characterization of polynomial transformations between vector spaces over a field of characteristic zero, 472.

Block, R. E. Differentiably simple algebras, 1086.

Bloom, C. O. Diffraction by a hyperbolic cylinder, 587.

Boardman, J. M. and Vogt, R. M. Homotopy-everything H-spaces, 1117.

Bragg, L. R. and Dettman, J. W. Related problems in partial differential equations, 375.

Brauer, Alfred and Gentry, I. C. On the characteristic roots of tournament matrices, 1133.

Brauer, George. Summability viewed as integration, 609.

Brauer, Richard, On simple groups of order $5 \cdot 3^{a} \cdot 2^{b}, 900$.

Brenner, J. L. Geř̌gorin theorems by Householder's proof, 625; erratum 768.

Browder, F. E. Nonlinear eigenvalue problems and Galerkin approximations, 651.

- Semicontractive and semiaccretive nonlinear mappings in Banach spaces, 660.

- and Nussbaum, R. D. The topological degree for noncompact nonlinear mappings in Banach spaces, 671.

$\longrightarrow$, and Petryskyn, W. V. The topological degree and Galerkin approximation for noncompact operators on Banach spaces, 641.

Brown, H. I. and Kerr, D. R. Some remarks of l-l summability, 529.

Bryant, J. L. and Seebeck, C. L., III. Locally nice embeddings in codimension three, 378.

Buck, R. C. Decidable semigroups, 892.

Burde, G. and Zieschang, H. A topological classification of certain 3-manifolds, 122.

Busenberg, Stavros. See Kraut Edgar. 
Butzer, P. L. and Görlich, E. Characterizations of Favard classes for functions of several variables, 149.

- and Trebels, W. Hilbert transforms, fractional integration and differentiation, 106.

- See Berens, $\mathrm{H}$.

Cantrell, J. C. and Lacher, R. C. Flattening a submanifold in codimensions one and two, 314.

Cashwell, E. D. and Everett, C. J. A general mean value theorem, 1135.

Chandra, Jagdish and Fleishman, B. A. Bounds and maximal solutions for nonlinear functional equations, 512.

Charnes, A. See Ben-Israel, A.

Cheeger, Jeff and Gromoll, Detlef. The structure of complete manifolds of nonnegative curvature, 1147.

Cholewinski, F. M. See Haimo, D. T.

Clay, J. R. See Anshel, Michael.

Coburn, L. A. See Berger, C. A.

Coifman, R. R. Remarks on weak type inequalities for operators commuting with translations, 710.

Connell, E. H. Examples of nontrivial H-cobordisms, 176.

Corner, A. L. S. and Crawley, Peter. An abelian p-group without the isomorphic refinement property, 743.

Cossey, John and Macdonald, S. O. A basis for the laws of PSL $(2,5), 602$.

Crawley, Peter and Hales, A. W. The structure of torsion abelian groups given by presentations, 954.

- See Corner, A. L. S.

Darst, R. B. On a theorem of Nikodym with applications to weak convergence and von Neumann algebras, 283.

Davis, W. J. Schauder decompositions in Banach spaces, 1083.

Dauns, John. Representation of $f$-rings, 249.

Della Riccia, Giacomo. Interiority of a holomorphic mapping on the set of its exceptional points, 484.

Dettman, J. W. See Bragg, L. R.

Diaz, J. B. and Margolis, Beatriz. A fixed point theorem of the alternative, for contractions on a generalized complete metric space, 305 .

Douglas, R. G. Toeplitz and Wiener-Hopf operators in $H^{\infty}+C, 895$.

Dunham, C. B. Continuity of the varisolvent Chebyshev operator, 606.

Duvall, P. F., Jr. Topological embeddings in codimension one, 1113.

Ebin, D. G. On the space of Riemannian metrics, 1001.

Ecker, J. G. See Peterson, E. L.

Edrei, Albert and Weitsman, Allen. Asymptotic behavior of meromorphic functions with extremal deficiencies, 140.

Elworthy, K. D. Fredholm maps and $\mathrm{GL}_{c}(E)$-structures, 582.

England, J. W. and Martin, N. F. G. On weak mixing metric automorphisms, 505.

Everett, C. J. See Cashwell, E. D.

Fabes, E. B. and Jodiet, M. Jr. $L^{p}$ boundary vabue problems for parabolic equations, 1098.

Farrell, F. T. and Hsiang, W. C. A geometric interpretation of the Kinneth formula for algebraic $K$-theory, 548.

Federer, Herbert. Some properties of distributions whose partial derivatives are representable by integration, 183. 
Feit, S. D. k-mersions of manifolds, 294.

Ferrar, J. C. See Allen, H. P.

Fields, Jerry. See Wimp, Jet.

Fisher, Stephen, The convex hull of the finite Blaschke products, 1128.

Flatto, Leopold. Basic sets of invariants for finite reflection groups, 730.

Fleishman, B. A. See Chandra, Jagdish.

Fogarty, J. and Rim, D. S. Serre sequences and Chern classes, 919.

Francis, G. K. The folded ribbon theorem for regular closed curves in the plane, 264.

Frank, D. L. An invariant for almost-closed manifolds, 562.

Freedman, D. A. On the derivative of a semigroup, 749.

Frolik, Zdeněk. Fixed points of maps of $\beta N, 187$.

Gentry, I. C. See Brauer, Alfred.

Gerstenhaber, Murray. The obstruction to an automorphism of a filtered ring, 695 .

Gilmer, Robert and Heinzer, William. On the existence of exceptional field extensions, 545.

Glassman, N. D. Cohomology of nonassociative algebras, 590.

Goldstein, L. J. Analogues of Artin's conjecture, 517.

Goodman, Roe. Analytic domination by fractional powers of a positive operator, 702.

Görlich, E. See Butzer, P. L.

Gretsky, N. E. Representation theorems on Banach function spaces, 705.

Grimm, L. J. and Schmitt, Klaus. Boundary value problems for delay-differential equations, 997.

Gromoll, Detlef. See Cheeger, Jeff.

Gross, Fred. On the equation $f^{n}+g^{n}=1$. II, 647.

, On the growth of $f(g), 1032$.

- Some theorems on factorization of meromorphic functions, 649.

Grossberg, Stephen. Global ratio limit theorems for some nonlinear functional-differential equations. I, 95.

- Global ratio limit theorems for some nonlinear functional-differential equations. II, 100.

Gupta, N. D. Polynilpotent groups of prime exponent, 559.

Gustafson, Karl. The angle of an operator and positive operator products, 488.

Haber, Seymour. A combination of Monte Carlo and classical methods for evaluating multiple integrals, 683.

Haimo, D. T. and Cholewinski, F. M. The Poisson-Laguerre transform, 137.

Hales, A. W. See Crawley, Peter.

Hall, William. See Kraut Edgar.

Hartman, Philip. Disconjugate nth order differential equations and principal solutions, 125.

Heinzer, William. See Gilmer, Robert.

Helgason, S. and Koranyi, A. A Fatou type theorem for harmonic functions on symmetric spaces, 258.

Hewitt, Edwin and Ross, K. A. A maximal problem in harmonic analysis. III, 225.

Hill, Paul. Extending automorphisms on primary groups, 1123.

Hilton, Peter and Roitberg, Joseph. Note on principal $S^{3}$-bundles, 957.

Hofmann, K. H. and Mostert P. S. The cohomology of compact abelian groups, 975.

Holland, S. S., Jr. On the adjoint of the product of operators, 931.

Hsiang, W. C. See Farrell, F. T.

Hubbuck, J. R. Some results in the theory of $H$-spaces, 965.

Jaco, William, Constructing 3-manifolds from group homomorphisms, 936. 
Jodiet, M. Jr. See Fabes, E. B.

Johnson, J. L. and Smoller, J. A. Global solutions of hyperbolic systems of conservation laws in two dependent variables, 915.

Jones, S. L. The impossibility of filling $E^{n}$ with arcs, 155.

Kamber, Franz. On the characteristic homomorphism of a discrete uniform subgroup of a nilpotent Lie group, 690.

Kato, Mitsuyoshi. Regular neighborhoods are not topologically invariant, 988.

Katz, Melvin. See Binmore, K. G.

Keller, H. B. Nonexistence and uniqueness of positive solutions of nonlinear eigenvalue problems, 887.

Kerr, D. R. See Brown, H. I.

Kirby, R. C. The union of flat $(n-1)$-balls is flat in $R^{n}, 614$.

Konheim, A. G. and Rivlin, T. J. The bounded linear operators that commute with the Bernstein operators, 111.

Korányi, A. See Helgason, S.

de Korvin, Expectations in von Neumann algebras, 912.

Kovács, L. G. Varieties of groups and Burnside's problem, 599.

-, Newman, M. F. and Pentony, P. F. Generating groups of nilpotent varieties, 968.

Kraut, Edgar, Busenberg, Stavros and Hall, William. On an additive decomposition of functions of several complex variables, 372.

Krengel, U. and Sucheston, L. On mixing in infinite measure spaces, 1150.

Kühn, Joachim. Ein Analogon zum high indices theorem für Potenzreihen mit wenigen vorzeichenwechseln, 133 ; errata, 628 .

Kwack, M. H. Generalization of the big Picard theorem, 759.

Lacher, R. C. Cell-like mappings of ANR's, 933.

See Cantrell, J. C.

Laha, R. G. Nonnegative eigen functions of Laplace-Beltrami operators on symmetric spaces, 167.

Landweber, P. S. Conjugations on complex manifolds and equivariant homotopy of $M U$, 271.

Lay, David. Characterizations of the essential spectrum of F. E. Browder, 246.

Lebow, Arnold. Maximal ideals in tensor products of Banach algebras, 1021.

Lickorish, W. B. R. and Martin, J. M. The impossibility of desuspending collapses, 979.

Lindsey, J. H., II. On a projective representation of the Hall-Janko group, 1094.

Loeb, P. A. and Walsh, Bertram. An axiomatic approach to the boundary theories of Wiener and Royden, 1004.

Lou, Betty. The centralizer of a regular unipotent element in a semisimple algebraic group, 1144.

Lucas, W. F. A game with no solution, 237.

Macdonald, I. G. Spherical functions on a p-adic Chevalley group, 520.

Macdonald, S. O. See Cossey, John.

Mallows, C. L. and Riordan, John. The inversion enumerator for labeled trees, 92.

Margolis, Beatriz. On some fixed points theorems in generalized complete metric spaces, 275.

- See Diaz, J. B.

Markin, J. T. A fixed point theorem for set valued mappings, 639.

Martin, D. A. The axiom of determinateness and reduction principles in the analytical hierarchy, 687. 
Martin, J. M. See Lickorish, W. B. R.

Martin, N. F. G. See England, J. W.

Masani, P. On the representation theorem of scattering, 618.

May, J. P. The cokomology of principal bundles, homogeneous spaces, and two-stage Postnikov systems, 334.

McClendon, J. F. A spectral sequence for classifying liftings in fiber space, 982.

McMillan, H. E. On the geometric theory of functions meromorphic in a disc, 119.

Michalicek, Johannes. See Arnold, Ludwig.

Miller, R. K. and Sell, G. R. A note on Volterra integral equations and topological dynamics, 904.

Mosher, R. E. Some homotopy of stunted complex projective space, 179.

Mostert, P. S. See Hoffmann, K. H.

Mowat, D. G. A Galois problem for mappings, 1095.

Mukherjea, K. K. Coincidence theory for infinite dimensional manifolds, 493.

Neggers, J. Some automorphism groups in Noetherian domains, 572.

Newman, B. B. Some results on one-relator groups, 568.

Newman, M. F. See Kovács, L. G.

Nussbaum, R. D. See Browder, F. E.

Oberst, Ulrich. Exactness of inverse limits, 1129.

Oddson, J. K. On the boundary point principle for elliptic equations in the plane, 666.

Orlik, P. and Rourke, C. P. Free involutions on homotopy $(4 k+3)$-spheres, 949.

Palis, J. On Morse-Smale diffeomorphisms, 985.

Palmer, T. W. Characterizations of $C^{*}$-algebras, 538.

Payne, S. E. A Bruck-Ryser type nonexistence theorem, 922.

Peck, N. T. A note on support points, 1103.

Pentony, P. F. See Kovács, L. G.

Peterson, E. L. and Ecker, J. G. A unified duality theory for quadratically constrained quadratic programs and $l_{p}$-constrained $l_{p}$-approximation problems, 316.

Pertyshyn, W. V. See Browder, F. E.

Poénaru, Valentin. Constructions on low-dimensional differentiable manifolds, 253.

Pollack, R. D. Restricted Lie algebras of bounded type, 326.

Putnam, C. R. Wiener-Hopf operators and absolutely continuous spectra. II, 340.

Rabin, M. O. Decidability of second order theories and automata on infinite trees, 1027.

Radjavi, Heydar and Rosenthal, Peter. Invariant subspaces and weakly closed algebras, 1013.

Rao, M. M. Local functionals and generalized random fields, 288.

Rauch, H. E. Functional independence of theta constants, 633.

Rhodes, John. The fundamental lemma of complexity for arbitrary finite semigroups, 1104 .

Richards, Ian. More on rings on rings, 677.

Rim, D. S. See Fogarty, J.

Riordan, John. See Mallows, C. L.

Rivière, N. M. Vector valued multipliers and applications, 946.

Rivlin, T. J. See Konheim, A. G.

Roitberg, Joseph. See Hilton, Peter.

Rolfsen, Dale. Strongly convex metrics in cells, 171.

Rosenthal, Peter. See Radjavi, Heydar.

Ross, K. A. See Hewitt, Edwin.

Rourke, C. P. See Orlik, P.

Rubel, L. A. See Berg. I. D. 
Rubin, Herman. Representation of nonnegative continuous functions on product spaces, 332.

Rubinstein, Zalman. On the approximation by C-polynomials, 1091.

Rudin, Walter. Modification sets of density zero, 526.

-, Proof of a conjecture of Helson, 727.

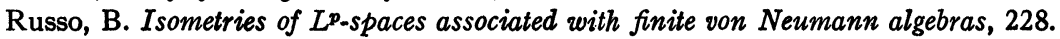

Schaeffer, A. J. Boundedness of solutions to linear differential equations, 508; errata 1036.

Schechter, Martin. Riesz operators and Fredholm perturbations, 1139.

Schmitt, Klaus, See Grimm, L. J.

Seebeck, C. L., III, See Bryant, J. L.

Sell, G. R. See Miller, R. K.

Shaneson, J. L. Embeddings with codimension two of spheres in spheres and H-cobordisms of $S^{1} \times S^{3}, 972$.

$\longrightarrow$ Wall's surgery obstruction groups for $Z \times G$, for suitable groups $G, 467$.

Shapiro, H. S. Some Tauberian theorems with applications to approximations theory, 500.

Sherman, T. L. On solutions of nth order linear differential equations with $N$ zeros, 923.

Shih, Weishu. On the symbol of a pseudo-differential operator, 657.

Shrader, Susan. See Wiginton, C. L.

Shult, Ernest. The solution of Boen's problem, 268.

Siebenmann, L. C. On the homotopy type of compact topological manifolds, 738.

Smith, J. W. An exact sequence for submersions, 233.

Smoller, J. A. See. Johnson, J. L.

Sotomayor, J. Generic one-parameter families of vector fields on two-dimensional manifolds, 722.

Stallings, John. Groups of dimension 1 are locally free, 361.

Stenger, William. On the projection of a self-adjoint operator, 369.

Stolzenberg, Gabriel. Constructive normalization of an algebraic variety, 595.

Strauss, Aaron and Yorke, J. A. Perturbing asymptotically stable differential equations, 992.

Sucheston, L. See Krengel, U.

Sullivan, F. E. Norm characterization of real $L^{p}$ spaces, 153.

Swan, R. G. Generators and relations for certain special linear groups, 576.

Swartz, Blair, $O\left(h^{2 n+2-l}\right)$ bounds on some spline interpolation errors, 1072.

Taylor, D. C. A characterization of Banach algebras with approximate unit, 761.

Trebels, W. See Butzer, P. L.

Vasquez, A. T. On characteristic classes of flat Riemannian manifolds, 715.

Verma, Daya-Nand. Structure of certain induced representations of complex semisimple Lie algebras, 160; errata 628.

Vogt, R. M. See Boardman, J. M.

Wales, D. B. Finite linear groups in seven variables, 197.

Wall, C. T. C. Free piecewise linear involutions on spheres, 554.

- Graded algebras, anti-involutions, simple groups and symmetric spaces, 198.

Walsh, Bertram. See Loeb, P. A.

Walsh, J. L. Note on classes of functions defined by integrated Lipshitz conditions, 344.

Warner, Seth. Two types of locally compact rings, 926.

Weidmann, Joachim. Strong Carleman operators are of Hilbert-Schmidt type, 735.

Weiss, N. J. Almost everywhere convergence of Poisson integrals on generalized halfplanes, 533. 
Weitsman, Allen. See Edrie, Albert.

Wells, G. S. Immersions of G-manifolds, $G$ finite, 130.

West, J. E. Extending certain transformation group actions in separable, infinite-dimensional Frechet spaces and the Hilbert cube, 1016.

Westphal, B. See Berens, H.

Wiegand, Roger. The cohomological dimension of Stone spaces, 944.

Wiginton, C. L. and Shrader, Susan. Spaces determined by a group of functions, 1010.

Wilf, H. S. Hadamard determinants, Möbius functions, and the chromatic number of a graph, 960.

Williams, F. D. A characterization of spaces with vanishing generalized higher White. head products, 497.

Wimp, Jet and Fields, Jerry. On the factorization of a class of difference operators, 1068.

Winter, D. J. Representations of locally finite groups, 145.

$\longrightarrow$, Solvable and nilpotent subalgebras of Lie algebras, 754.

Wong, J. S. W. Second order linear oscillation with integrable coefficients, 909.

Wong, Y.-C. Conjugate loci in Grassmann manifolds, 240.

Wright, Perrin. A uniform generalized Schoenflies theorem, 718.

Yorke, J. A. Extending Liapunov's second method to non-Lipshitz Liapunov functions, 322.

See Strauss, Aaron.

Youngs, J. W. T. Remarks on the Heawood conjecture (nonorientable case), 347. , The nonorientable genus of $K_{n}, 354$.

Zieschang, H. See Burde, G.

\section{BOOK REVIEWS}

Bauer, Heinz. Review of "Probability and potentials," by P. A. Meyer, 75.

Shapiro, V. L. Review of "Real and complex analysis," by Walter Rudin, 79.

\section{REPORTS OF MEETINGS AND MISCELLANEOUS ARTICLES}

Anderson, R. D. and Bing, R. H. A complete elementary proof that Hilbert space is homeomorphic to the countable infinite product of lines, 771.

Bateman, P. T. Reports of Meetings of the American Mathematical Society: The November meeting in Urbana, 91.

- See Pitcher, Everett.

Bing, R. H. See Anderson, R. D.

Council and Board of Trustees-1967, 204.

Federer, Herbert. Reports of Meetings of the American Mathematical Society: The October meeting in Cambridge, 89; The April Meeting in New York, 1066.

Harrold, O. G. Reports of Meetings of the American Mathematical Society: The November meeting in Knoxville, 90.

Martin, W. T. Reports of the Treasurer, 1966 and 1967, 85, 884.

Peirce, R. S. Reports of Meetings of the American Mathematical Society: The March meeting in Los Angeles, 631; The November Meeting in Albuquerque, 224; The June Meeting in Portland, 1067.

- See Pitcher, Everett.

Pitcher, Everett, 1967 Election of Council Members and Board of Trustees, 84.

, and Bateman, P. T. Reports of Meetings of the American Mathematical Society: The April Meeting in Chicago, 880. 
and Pierce, R.S. Reports of Meetings of the American Mathematical Society: The Annual meeting in San Francisco, 455.

Thompson, J. G. Nonsolvable groups all of whose local subgroups are solvable, 383.

\section{INVITED ADDRESSES}

Albert, A. A. On associative division algebras, 438. 455.

Almgren, F. J. 1066.

Atkinson, F. V. Multi-parameter spectral theory, 1.

Auslander, Louis, 455.

Bade, W. G. 1067.

Brown, E. H. 89.

Gale, David. A mathematical theory of optimal economic development, 207.

Getoor, R. K., 224.

Goodman, A. W. Problems on univalent and multivalent functions, 1035.

Gordon, Basil, 224.

Heller, Alex. Stable homotopy categories, 28.

Householder, A. S., 90. , Norms and the localization of roots of matrices, 816.

Jonsson, Bjarni, 90.

Lefshetz, Solomon. A page of mathematical autobiography, 854.

Lewis, D. J., 91.

Luxemburg, W. A. J., 1067.

Mahowald, M. E., 91.

Mattuck, A. P., 89

McKean, H. P., 1066.

Peterson, F. P., 1066.

Porcelli, Pasquale, 90.

Ree, Rimhak, 631.

Reiner, Irving, 880.

Seligman, G. B., 1066.

$\longrightarrow$, Algebraic Lie algebras, 1051.

Smale, Stephen, 880.

Thom, Rene, 880 .

Tutte, W. T., On the enumeration of planar maps, 64.

Wasow, Wolfgang, 455.

, Connection problems for asymptotic series, 831 .

Weinberger, Hans, 880.

Wigner, E. P., 455.

—, Symmetry principles in old and new physics, 793. 